\title{
Nanotechnology in Head and Neck Cancer: The Race Is On
}

\author{
Ivan H. El-Sayed
}

Published online: 3 March 2010

(C) The Author(s) 2010. This article is published with open access at Springerlink.com

\begin{abstract}
Rapid advances in the ability to produce nanoparticles of uniform size, shape, and composition have started a revolution in the sciences. Nano-sized structures herald innovative technology with a wide range of potential therapeutic and diagnostic applications. More than 1000 nanostructures have been reported, many with potential medical applications, such as metallic-, dielectric-, magnetic-, liposomal-, and carbon-based structures. Of these, noble metallic nanoparticles are generating significant interest because of their multifunctional capacity for novel methods of laboratory-based diagnostics, in vivo clinical diagnostic imaging, and therapeutic treatments. This review focuses on recent advances in the applications of nanotechnology in head and neck cancer, with special emphasis on the particularly promising plasmonic gold nanotechnology.
\end{abstract}

Keywords Nanotechnology · Gold nanoparticles .

Nanorods $\cdot$ Cancer $\cdot$ Head and neck

\section{Introduction}

Nanobiotechnology represents the convergence of multiple scientific fields including chemistry, engineering, physics, and molecular biology. Nanoparticles are generating a revolution within the scientific community as the race to develop clinically useful structures progresses. Elements

I. H. El-Sayed ( $\bowtie)$

Otolaryngology Minimally Invasive Skull Base Program,

Department of Otolaryngology-Head and Neck Surgery,

Department of Neurologic Surgery, University of California,

San Francisco,

2380 Sutter Street, 3rd Floor,

San Francisco, CA 94115, USA

e-mail: ielsayed@ohns.ucsf.edu restricted to the nanoscale in various forms demonstrate previously unseen physical properties (electronic, optical, magnetic, catalytic) [1]. Due the appropriate size match and facile surface chemistry allowing conjugation to biologically active molecules, several nanoparticles are potentially exploitable for a wide range of applications in biology and medicine. Nanotechnology has generated significant investment from the National Institutes of Health (NIH)/National Cancer Institute and is expected to create a paradigm-shift in the detection, treatment, and prevention of cancer [2].

A plethora of nanostructures have been described with varying composition (eg, gold, iron oxide, carbon, dielectric materials, molecular, liposomal) and shapes including solid nanoparticles (eg, spheres, rods, triangles, cubes), nanoshells (within inner and outer cores), nanocages, nanowire, nanotubes, branched dendrimers, and polymeric and organic lipid nanoparticles [3]. Inorganic nanoparticles are particularly interesting because of their unique electronic, magnetic, optical, photothermal, or catalytic properties at the nanoscale. Of the array of nanostructures currently available, plasmonic gold nanoparticles are especially promising because of their simple fabrication, multifunctional nature, facile surface chemistry $[3-6 \cdot \bullet]$, biodistribution properties, and relatively low toxicity $[7 \cdot, 8,9]$.

Gold nanoparticles can be applied therapeutically for photothermal therapy [3], intravascular drug/gene delivery [10], and ionizing radiation enhancement [11]. This new technology has impending clinical application toward head and neck cancer as evidenced by the initiation of two phase 1 human trials investigating gold conjugated tumor necrosis factor (TNF) treatment of solid tumors [12] and photothermal therapy of refractory head and neck cancer [13]. A discussion of gold nanotechnology provides a foundation to understand the unique possibilities created by the emerging nanosciences. 


\section{Why Nanobiotechnology?}

Nanotechnology is expected to provide a range of devices for diagnosis and treatment in medicine. On the size scale of 1 to $200 \mathrm{~nm}$, nanoparticles are well matched in size to biologic molecules and structures found inside living cells [14]. Typical mammalian cells range in size from $2000 \mathrm{~nm}$ to $10,000 \mathrm{~nm}$, whereas cellular organelles are about $100 \mathrm{~nm}$ to $300 \mathrm{~nm}$ and intracellular proteins and molecules are about $10 \mathrm{~nm}$ to $50 \mathrm{~nm}$. Nanoparticles appear the appropriate size range for imaging and manipulation at the molecular level. The ability to control the nanoparticle surface chemistry allows conjugation to various ligands for interactions at the molecular level. Furthermore, gold nanoparticles produce greatly enhanced spectral signals that are detectable above the background noise of cells and extracellular tissue.

\section{Head and Neck Cancer: Room for Improvement}

Cancer is the second-leading cause of mortality in the United States, responsible for one in every four deaths [15]. Cancers of the head and neck-including the salivary glands, thyroid, and the mucosal lining of the oral cavity, pharynx, nasopharynx, and larynx - account for $2 \%$ to $6 \%$ of all malignancies in the United States [16]. The mainstay of treatment for head and neck cancer is surgery, radiation, chemotherapy, antibody-blocking therapy, or a combination of therapies. The most important advance in the management of head and neck cancer has been the increasing role of chemotherapy, or antibody-blocking therapy, for use in conjunction with radiation therapy to achieve cure with organ preservation $[17,18]$. Despite these advances, survival rates for head and neck cancer have improved little in the past 50 years. Current treatments have significant functional and cosmetic impact and significant attention is focused on improving the quality of life of these patients [17].

Oral squamous cell carcinoma (OSCC) represents an excellent model for both head and neck cancer and solid malignancies in general. Nearly $85 \%$ of all malignancies are of epithelial origin including the skin, oral cavity, nasopharyngeal, laryngeal, lung, gastrointestinal, colon, and bladder cancer [15]. OSCC is an aggressive malignancy that invades local tissue, spreads to regional and distant sites, and has an overall 5-year survival of $60 \%$ [15]. About $80 \%$ to $90 \%$ of OSCC overexpress a surface antigen important to tumor growth and proliferation, epithelial growth factor receptor (EGFR) [19]. Analogous surface receptors exist on other solid tumors, making EGFR an excellent model for investigation of antibody-based targeting of tumors. EGFR-blocking therapies have enjoyed recent success as adjuvant treatment to radiation with significant reduction in associated toxicity for patients [18]. Detection of OSCC primary or recurrent tumors is possible with direct visual inspection because of surfaceexposed location in the body. As a result of these factors, OSCC has been the focus of several early studies of nanotechnology.

\section{Find a Role for Nanotechnology in Cancer Management?}

Nanotechnology appears poised to provide devices capable of 1) sensitive and specific anatomic, molecular, and biologic imaging; 2) selective therapy of tumors; and 3) relatively low toxicity. The physical properties of several nanostructures strongly suggest that these goals are attainable and will result in a significant improvement over the current standard of care. Patients with head and neck cancer require staging assessments, invasive treatments, and posttreatment monitoring with physical examination and routine imaging for 5 years. The mainstay of imaging modalities for diagnosis and follow-up head and neck cancer patients are MRI, CT, ultrasonography, and positron emission tomography (PET). These techniques have limited resolution and cannot detect microscopic or molecular changes. Further, interpretation of findings can be complicated by difficult anatomy, edema or inflammation, scarring from prior treatment, and loss of detail because of patient movement or dental implants. False-positive findings can occur on PET imaging because of inflammatory or infectious processes. Furthermore, the imaging techniques are quite poor for detection of small surface lesions. Definitive diagnosis still requires tissue diagnosis with biopsy or needle aspiration. Intraoperative diagnosis of tumor at the surgical margins can be difficult. Developing sensitive and specific noninvasive molecular tests for staging, screening, and intraoperative diagnosis would significantly improve patient care.

Currently available therapies for head and neck cancer suffer significant limitations. In the head and neck, surgical resection is limited by several adjacent important structures such as the carotid artery, eye, and brain. Residual tumor may be left behind near vital structures or because it has spread beyond the surgical margin, making adjuvant treatments necessary to achieve cure of the residual disease. Radiation therapy has a high failure rate for advanced tumors, and toxicity limits the amount that can be given to one full course treatment. Chemotherapy in head and neck cancer is limited to a supportive role in combination with radiation. Nanotechnology may provide a new tool for clinicians by offering the potential of molecular diagnostic probes and novel therapeutic devices, such as photothermal and magneto-thermal probes, drug- and gene-delivery vectors, and radiation enhancers. 


\section{Application of Gold Nanotechnology in Head and Neck Cancer}

Three properties of gold nanoparticles are important at the biologic level. First, the small size of the particles creates a large surface area to volume. Based on the large surface area, chemical reactions occur on the surface of the nanoparticles at a significantly increased rate, creating actively enhanced catalytic agents. The catalytic properties have not yet found application in cancer therapy. Second, nanoparticles happen to be the correct size for intravascular transport and accumulation inside many tumor beds for selective tumor targeting and drug delivery. Third, gold particles take on optical properties useful for imaging and photothermal therapy.

\section{Gold Nanoparticles as a Drug-Delivery Vector}

When hidden from the human immune system, gold nanoparticles ranging from $10 \mathrm{~nm}$ to $140 \mathrm{~nm}$ will accumulate inside tumors because of the enhanced permeability and retention effect [20]. Passivating the nanoparticle surface can be easily achieved with polymers such as polyethylene glycol (PEG). Accumulation in solid tumors occurs because of filtration through the poorly formed, leaky vasculature associated with tumor angiogenesis [20]. Paciotti et al. [8] demonstrated PEG bound gold conjugated with TNF rapidly accumulated in MC-38 colon carcinoma tumor-bearing mice with little uptake in other organs [8]. The PEG coating shields the complex from the immune responses and is vital for successful drug delivery. Paciotti et al. [8] discovered gold nanoparticles with targeting ligands lacking a passivating agent are taken up within minutes by the reticuloendothelial system. Because gold readily bonds to sulfur atoms, gold nanoparticles can be easily conjugated to a range of molecules through thiol chemistry. Loading gold with toxic chemicals or molecules, such as TNF, allows selective delivery of large doses of toxins. By adding PEG in addition to selected toxins, selective accumulation in the tumor reduces toxicity of the treatment drug by avoiding the other healthy host organs. Interestingly, the targeting ligand TNF appears to increase uptake of the gold into the tumor, suggesting that active ligands can improve selective tumor targeting. As a result of these findings, gold-based drug delivery is the first NIH funded human "nano" trial initiated and has completed patient accumulation in its phase 1 trial [21]. Several potential targeting ligands exist to improve tumor uptake including folate, transferrin, arginine-glycine-aspartic acid peptide, antibodies, or antibody fragments to cell surface receptors [22].

\section{Optical Properties of Gold Useful for Imaging and Therapy}

\section{Plasmons}

Noble metal nanoparticles such as solid gold, silver, and silica-core gold nanoshells exhibit dramatic optical properties useful for ultrasensitive sensing and photothermal therapy. When stimulated by light, all the conduction band electrons in the gold nanoparticles oscillate coherently on the particle surface, creating a phenomena known as the surface plasmon resonance (SPR). According to the Mie theory, when light strikes gold nanoparticles smaller than $200 \mathrm{~nm}$, energy is lost due to two processes: light scattering and light absorption [6].

Light scattering occurs as energy from the light stimulates the electrons on the particle surface to oscillate and subsequently emit photons at the same frequency (color) or at a shifted frequency. When light is absorbed, the energy is converted efficiently into heat. The nanoparticles have a particular (resonance) frequency, or color, in which they scatter and absorb the maximum amount of light. The "peak frequency" is sensitive to the size, shape, composition and surrounding environment. By changing the size or shape, gold nanoparticles are tunable over the visible and infrared region of light. Particles $30 \mathrm{~nm}$ to $100 \mathrm{~nm}$ in size scatter intensely and can be detected by commercial microscopes with darkfield illumination. Using a darkfield microscope, particles $40 \mathrm{~nm}$ in size can be detected by eye at a concentration of $10^{-14} \mathrm{M}$. A single $60 \mathrm{~nm}$ nanosphere is $10^{5}$ times brighter than a fluorescein molecule $[3,23]$. Changing the electric field of the nanoparticles, such as by bringing two particles in close proximity to each other, changes the peak frequency by shifting the color toward the red region. A few nanoparticles will cause a shift of a few nanometers, whereas aggregation of several particles causes a larger red shift of up to $100 \mathrm{~nm}$. If the nanoparticle is coated with a substance, such as an antibody, the light must pass through that substance, resulting in a small red shift of the peak frequency. The attributes are useful for plasmonic based imaging [3].

\section{Plasmonic Cancer Imaging}

In addition to direct interaction with light, the SPR creates a source of energy on the particle surface that can affect optical properties of molecules on its surface. Gold nanotechnology appears useful for biomedical imaging in many optical domains including fluorescence [24], light absorption and scattering $[4,25,26]$, photoacoustic imaging [27], photothermal imaging [28], and surfaceenhanced Raman scattering [29]. Based on the SPR, diagnostic imaging with gold nanospheres and nanorods 
has been achieved in vivo in animal models using multiphoton imaging of single nanorods [30], photoacoustic imaging of tumors and sentinel nodes [31, 32], near infrared absorption imaging [7•, 33], surface-enhanced Raman spectroscopy [34・•], and confocal endoscopy [35].

As optical sensors, gold nanoparticles are multifunctional probes that provide several new types of information for medical imaging at the subcellular, biochemical, and molecular level. Silica-gold nanoshells and solid gold nanoparticles, as spheres and rods, can be selectively targeted to OSCC using antibodies directed against EGFR. Sokolov et al. [26] demonstrated in vitro immunoconjugated gold nanospheres are intense contrast agents using a red laser pointer with a portable confocal endoscopy unit. El-Sayed et al. [25] demonstrated the colorimetric attributes of immunotargeted gold nanoparticles for imaging anatomic location and molecular sensing of OSCC with light scattering and absorption. Imaging of gold nanospheres revealed colored particles with much greater affinity for the malignant cell lines. Particles about $25 \mathrm{~nm}$ in size could easily be detected above the background scattering of the cellular components. Light absorption was measured from single cells revealing characteristic absorption spectra of the gold nanospheres. The authors reported measurement of a shift in the color frequency by approximately $10 \mathrm{~nm}$ when the antibody-gold conjugated bound the EGFR. Furthermore, the amount of nanoparticles on the cell surface could be quantified as binding to the malignant cell line is $600 \%$ more than to the control cells, consistent with expected values of overexpression of EGFR on the cell lines [25]. Huang et al. [4] subsequently demonstrated gold nanorods as near-infrared (NIR) imaging probes in the same OSCC cell line model using light scattering and absorption. Plasmonic imaging was subsequently performed in vivo using light scattering. The authors found about a $100 \mathrm{~nm}$ red shift of the particles color (from green to red) on the cell surface. The imaging characteristics and color change are thought to be caused by the aggregation of the nanoparticles induced by the clustering of overexpressed EGFR on the cancer cell surface.

\section{Fluorescence Imaging of Gold Nanoparticles}

The SPR can further enhance other optical processes of molecules in close proximity to the nanoparticles. For instance, gold nanoparticles quench fluorescence of molecules when covalently bound to the molecule, but enhance the scattering and fluorescence of molecules on their surface is separated from the nanoparticles by a distance sufficient to minimize quenching. In OSCC cell culture models, El-Sayed et al. [24] found that gold nanoparticles quench cellular autofluorescence approximately $15 \%$ when incubated or immunoconjugated to cells. This effect was interpreted to be due to intense light absorption of the particles that were restricted in cellular compartments from accessing strong cellular fluorophores.

Molecular Detection with Surface-enhanced Raman Scattering

Surface-enhanced Raman scattering (SERS) is a powerful technology potentially useful for single-cell study and clinical medical diagnostics in vivo. Raman scattering of light occurs as a result of modulating the scattered light by the vibrational frequencies of the irradiated molecules. This produces a so-called fingerprint spectrum specific to the molecule. Because of the typically weak signals, use of Raman spectroscopy has been limited in medical applications. However, the gold SPR strongly enhances Raman scattering of adjacent molecules, creating a surfaceenhanced Raman spectroscopy, known as SERS. Gold SPR enhances the Raman scattering by orders of magnitude (more than a million times) [34] and has been reported for the use of SERS for single molecule detection [36] and in vivo detection of cancer in a murine model. Gold nanospheres tagged with colored dyes and covalently bound to anti-EGFR single chain variable fragment $(\mathrm{Sc}-\mathrm{Fv}) \mathrm{B} 10$ antibodies produced strong SERS spectra of the dyes using a $20 \mathrm{~mW}$ laser at $9 \mathrm{~mm}$ from the skin. The dyes were 200 times brighter than the fluorescence emitted by the comparison group labeled with quantum dots [34]. Furthermore, SERS spectra have been obtained from nanoparticles targeted to the cytoplasm, nucleus [37], and cell surface [38]. Huang et al. [38] identified a possible molecular signature of EGFR overexpression using antibodyconjugated gold nanorods. The anti-EGFR nanorod SERS produced a strong, polarized spectra. The great intensity of signals created by gold nanorods results from the overlapping gold plasmonic fields of the rods lined lengthwise that would not occur with haphazard alignment on the cell surface. The lengthwise arrangement of the nanorods is postulated to result from tight packing of some of the rods in close proximity because of overexpression, clustering, and dimerization of EGFR receptors on the cancer cell surface [38].

\section{Plasmonic Photothermal Therapy}

Another useful application of light absorption by gold nanoparticles is generation of heat for plasmonic photothermal therapy (PPTT). By changing the size, shape, or composition of the nanoparticles, the color of light that absorbs maximal energy can be tuned over the visible and NIR spectra. The NIR region from $650 \mathrm{~nm}$ to $900 \mathrm{~nm}$ is a desirable optical window in human tissue for deep 
penetration of light. Whereas gold spheres are tunable across the visible spectrum, the nanoshells and nanorods are both tunable across the NIR spectrum. The main determining factor of nanorod peak absorption is the aspect ratio of the particle: the relation of the length to width. By changing the aspect ratio (ratio of the short end to long end), gold nanorods are tunable over the near IR region from $650 \mathrm{~nm}$ to $1000 \mathrm{~nm}$, where tissue penetration of human tissue by light is maximal. Although light penetration of human tissue is limited to a few millimeters in the visible region, microwatt laser NIR light can penetrate $10 \mathrm{~cm}$ into breast tissue and $4 \mathrm{~cm}$ into skull/ brain or deep muscle tissue. Higher power US Food and Drug Administration class 3 lasers can penetrate through $7 \mathrm{~cm}$ of muscle and neonatal skull/brain [39]. From a surgeon's perspective, in practical use, light delivery and nanoparticle selection can be tailored to specific lesions. Light and nanoparticles can be delivered externally, within the tumor by intravascular canalization, direct needleguided placement, or into a postoperative field for residual tumor cells.

Gold nanoshells, a first generation nanotechnology with a silica core surrounded by a gold shell, have been successfully tested in vitro [40,41], in vivo in animal models [42], and are currently initiating human trials for NIR photothermal therapy in refractory head and neck cancers [13]. However, based on modeling of heating efficiencies of gold nanospheres, nanorods, nanoshells and molecular dyes, gold nanorods seem to be far superior agents [43].

Selective targeting with PPTT of OSCC was demonstrated in vitro in the visible range with immunoconjugated gold nanospheres [5]. Using a laser in the visible region at $442 \mathrm{~nm}$, gold nanospheres were efficiently heated and killed the cells in two malignant OSCC cell lines at $19 \mathrm{~W} / \mathrm{cm}^{2}$ (HOC 313 clone 8 ) and $25 \mathrm{~W} / \mathrm{cm}^{2}$ (HSC 3) at much lower energy compared to the nonmalignant cell line (HaCat) at $57 \mathrm{~W} / \mathrm{cm}^{2}$ [5]. However, visible light only penetrates human tissue on the order of a few millimeters and nanospheres would be limited to surface applications.

By changing the shape to a rod, or using a nanoshell, the peak absorption wavelength can be tuned to the NIR spectrum. Gold nanoshells targeted to breast cancer [41] and gold nanorods targeted to OSCC in the NIR [4] have been reported for combined imaging and photothermal therapy at $800 \mathrm{~nm}$ using a Ti:sapphire laser [4]. Nanorods achieve tumoricidal effect with less energy than either nanoshells or nanospheres. Nanoshells caused cell death at $35 \mathrm{~W} / \mathrm{cm}^{2}$ for $7 \mathrm{~min}$ exposure [41], whereas immunotargeted gold nanorods achieved cell death with $3 \mathrm{~min}$ exposure at $10 \mathrm{~W} / \mathrm{cm}^{2}$ in vitro [7].

Based on heating and modeling experiments, gold nanoparticles can generate temperatures of $70^{\circ} \mathrm{C}$ to $80^{\circ} \mathrm{C}$ in these cells using far lower laser powers than conventional dyes [44]. Temperatures of $70^{\circ} \mathrm{C}$ to $80^{\circ} \mathrm{C}$ celsius are hot enough to denature proteins and disrupt protein, DNA, and RNA. Image analysis suggest cell death occurs because of blebbing formation of the cell wall and loss of membrane integrity [45]. Cell injury is likely related to both necrosis and cell membrane rupture.

Comparison of gold nanospheres, nanorods, and nanoshells reveals the gold nanoparticles absorb light at $10^{4}$ times better than the best molecular dyes. Gold nanorods absorb a similar amount of energy as gold nanoshells at one third the size because the entire nanorod is composed of gold [43]. Furthermore, nanorods absorb the most energy per particle volume of all the particles. To compare particles across a range of sizes, a volumetric coefficient, expressed as a per micron absorption coefficient $u_{\mathrm{abs}}$ can be calculated. Examining the nanoshell configuration used in vivo for photothermal trials (inner core, $60 \mathrm{~nm}$; outer core, $70 \mathrm{~nm}$ ) [40, 41], the nanoshells have a $u_{\text {abs }}$ of 35.62 with a peak absorption at $892 \mathrm{~nm}$. At $11.4 \mathrm{~nm}$, the nanorods $u_{\text {abs }}=$ 1000.87 at $863 \mathrm{~nm}$, roughly 30 times more than the nanoshells (Table 1) [43].

Both nanorods and nanoshells are tested in vivo with PPTT. Hirsch et al. [41] found a maximal temperature rise of $37^{\circ} \mathrm{C}$ using 4 to $6 \mathrm{~min}$ of laser exposure in a murine model of transmissible venereal tumor with 10 - to 25 -fold less laser energy than needed for indocyanine green dye [41]. In an OSCC murine model, PEGylated nanorods were successfully imaged in OSCC implanted murine tumors using an infrared light source and charge coupled device

Table 1 Comparison of absorption coefficient of nanorods and nanoshells

\begin{tabular}{|c|c|c|c|}
\hline Particle $^{\mathrm{a}}$ & Dimension & $\lambda_{\max } \mathrm{nm}$ & $\mu_{\mathrm{a}}$ \\
\hline Silica-gold & $\begin{array}{l}\mathrm{R} 1=40 \mathrm{~nm} \\
\mathrm{R} 2=70 \mathrm{~nm}\end{array}$ & 843 & 50.61 \\
\hline Silica-gold & $\begin{array}{l}\mathrm{R} 1=50 \mathrm{~nm} \\
\mathrm{R} 2=70 \mathrm{~nm}\end{array}$ & 704 & 20.57 \\
\hline Silica-gold & $\begin{array}{l}\mathrm{R} 1=90 \mathrm{~nm} \\
\mathrm{R} 2=105 \mathrm{~nm}\end{array}$ & 984 & 11.07 \\
\hline Nanorods & $\mathrm{AR}=3.1, \mathrm{r}_{\mathrm{eff}}=11.43 \mathrm{~nm}$ & 797 & 907.09 \\
\hline Nanorods & $\mathrm{AR}=3.9, \mathrm{r}_{\mathrm{eff}}=11.43 \mathrm{~nm}$ & 863 & 1003.87 \\
\hline Nanorods & $\mathrm{AR}=3.9, \mathrm{r}_{\mathrm{eff}}=8.74 \mathrm{~nm}$ & 788 & 986 \\
\hline Nanorods & $\mathrm{AR}=3.9, \mathrm{r}_{\mathrm{eff}}=21.86 \mathrm{~nm}$ & 842 & 449.34 \\
\hline
\end{tabular}

${ }^{\text {a }}$ Gold nanorods have about 30 times more absorption of light in the size range of particles under study for photothermal applications.

$A R$ aspect ratio; $\lambda_{\max }$ wavelength where particle has maximal absorption and scattering (extinction); $\mu_{a}$ per micron absorption coefficient; $R_{1}$ outer gold core; $R_{2}$ inner silica core; $r_{\text {eff }}$ effective radius of nanorod.

(Adapted from Jain et al. [43], with permission.) 
digital camera (Fig. 1). Treatment with an $808 \mathrm{~nm}$ LED laser with a $6 \mathrm{~mm}$ beam achieved a $22^{\circ} \mathrm{C}$ temperature rise and a $25 \%$ (intravenous route) and $57 \%$ (direct injection into tumor) reduction of tumor compared with the sham treatment group after 13 days. Imaging revealed the direct injection group had 2.18 times greater absorption of light in the tumor than the intravenous injection group and 4.35 greater than the control group at 2 min (Fig. 1).

The biodistribution of gold nanorods appears similar to other nanoparticles and can be delivered intravascularly to tumors. In vivo mice studies demonstrated PEG-nanorods accumulates about one third of the gold in the tumor [9, 46], making gold nanorods very attractive for PPTT applications in the head and neck.

\section{Radiation Enhancement}

Gold nanotechnology is potentially useful to augment current ionizing radiation techniques through two avenues: tissue hyperthermia and radiation enhancement. Gold is strong absorber of radiographs. Hainfeld et al. [47] calculated that radiation doses could be enhanced locally around gold nanoparticles loaded into tumors by more than $200 \%$. Tumor-burdened mice treated with gold nanoparticles and radiation demonstrated 86\% 1-year

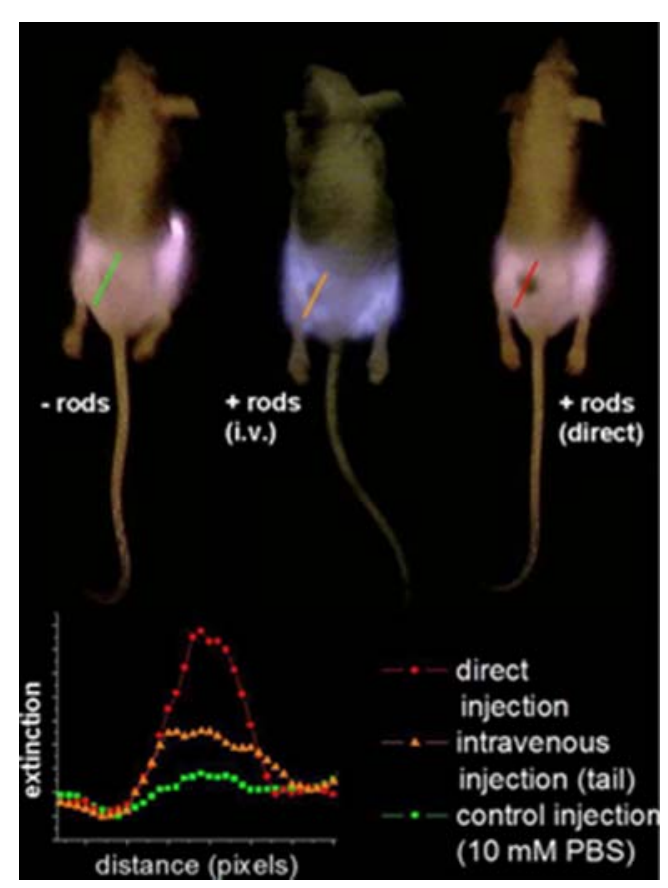

Fig. 1 Near infrared absorption imaging with gold nanorods in mice treated with tumors (top). Extinction spectra (bottom) reveal that direct injection achieves nearly twice the absorption of intravascular injection of the nanorods. PBS - phosphate buffered saline. (From Dickerson et al. [7•], with permission.) survival in gold nanoparticle treatment group compared with only $20 \%$ in group treated with radiation alone [11]. In addition, the effectiveness of ionizing radiation can be improved with local tissue hyperthermia during treatment. This effect can be achieved using photothermal techniques [48].

\section{Other Technologies}

Other nanotechnologies clearly have significant potential for application in the diagnosis and management of head and neck cancer, such as carbon nanotubes, iron oxide (magnetic) nanoparticles, and dendrimers [2, 22, 49]. For instance, iron-based nanoparticles work on similar principles to gold nanoparticles as magnetically active probes for imaging, therapy, and drug carrying [49]. A variety of additional ligands have been attached to nanoparticles to selectively target cancer cells. Other classes of nanoparticles can serve as drug or gene carries. As later generations of nanotechnology develop, such as combined magnetico-optico particles [50], integrated particles comprising "nanosystems" are expected to be developed capable of performing complex actions.

\section{Conclusions}

Nanotechnology is poised to create a paradigm shift in the diagnosis and management of head and neck cancer through the creation of multifunctional devices capable of sensitive, specific diagnosis and simultaneous therapy. Whereas several nanoparticles have been developed, plasmonic gold nanoparticles appear particularly interesting because of their facile surface chemistry, relatively limited toxicity, and novel optical properties useful for concurrent imaging and therapy. Gold nanotechnology brings forth ultrasensitive optical imaging and multiple therapeutic options that may be potentially used in unison. As a drug delivery agent, toxicity of attached drugs is significantly reduced. Photothermal therapy adds a new treatment that can be used in addition to other forms of treatment. Enhancement of radiation could significantly improve delivery of appropriate radiation doses with reduced toxicity to surrounding tissue. Although much work is still required to understand the toxicity and best applications, compared with the limitations and toxicities of existing treatment, nanotechnology may significantly advance management of head and neck cancer.

Disclosure No potential conflicts of interest relevant to this article were reported. 
Open Access This article is distributed under the terms of the Creative Commons Attribution Noncommercial License which permits any noncommercial use, distribution, and reproduction in any medium, provided the original author(s) and source are credited.

\section{References}

Papers of particular interest, published recently, have been highlighted as:

- Of importance

- Of major importance

1. El-Sayed MA: Some interesting properties of metals confined in time and nanometer space of different shapes. Acc Chem Res 2001, 34:257-264.

2. Pridgen EM, Langer R, Farokhzad OC: Biodegradable, polymeric nanoparticle delivery systems for cancer therapy. Nanomed 2007, 2:669-680.

3. Huang X, Jain PK, El-Sayed IH, El-Sayed MA: Gold nanoparticles: interesting optical properties and recent applications in cancer diagnostics and therapy. Nanomed 2007, 2:681-693.

4. Huang X, El-Sayed IH, Qian W, El-Sayed MA: Cancer cell imaging and photothermal therapy in the near-infrared region by using gold nanorods. J Am Chem Soc 2006, 128:2115-2120.

5. El-Sayed IH, Huang X, El-Sayed MA: Selective laser photothermal therapy of epithelial carcinoma using anti-EGFR antibody conjugated gold nanoparticles. Cancer Lett 2006, 239:129-135.

6. •• Jain PK, El-Sayed IH, El-Sayed MA: Au nanoparticles target cancer. NanoToday 2007, 2:18-29. This article presents a thorough review of the optical properties of gold nanoparticles useful for imaging and treatment of cancer.

7. •Dickerson EB, Dreaden EC, Huang X, et al.: Gold nanorod assisted near-infrared plasmonic photothermal therapy (PPTT) of squamous cell carcinoma in mice. Cancer Lett 2008, 269:57-66.

8. Paciotti GF, Kingston DG, Tamarkin L: Colloidal gold nanoparticles: a novel nanoparticle platform for developing multifunctional tumortargeted drug delivery vectors. Drug Dev Res 2006, 67:47-54.

9. Niidome T, Yamagata M, Okamoto Y, et al.: PEG-modified gold nanorods with a stealth character for in vivo applications. J Control Release 2006, 114:343-347

10. Paciotti GF, Myer L, Weinreich D, et al.: Colloidal gold: a novel nanoparticle vector for tumor directed drug delivery. Drug Deliv 2004, 11:169-183.

11. Hainfeld JF, Slatkin DN, Smilowitz HM: The use of gold nanoparticles to enhance radiotherapy in mice. Phys Med Biol 2004, 49:N309-N315.

12. Clinicaltrials.gov: TNF-bound colloidal gold in treating patients with advanced solid tumors, in, 2010. Available at http://clinicaltrials.gov/ ct2/show/NCT00356980. Accessed January 2010.

13. Clinicaltrials.gov: Pilot study of AuroLase(tm) therapy in refractory and/or recurrent tumors of the head and neck, in, National Institute of Health, 2010. Available at http://clinicaltrials.gov/ct2/ show/NCT00848042. Accessed January 2010.

14. Whitesides GM: The "right" size in nanobiotechnology. Nat Biotechnol 2003, 21:1161-1165.

15. American Cancer Society: Cancer Facts and Figures, 2009. Available at http://www.cancer.org/downloads/STT/500809web.pdf. Accessed Januatry 2010.

16. Myers EN, Simental AA: Cancer of the oral cavity. In Cancer of the Head and Neck. Edited by Myers EN, Suen JY, Myers JN, Hanna EY. Philadelphia, PA: Saunders; 2003:279-332.
17. Myers EN, Suen JY: Perspectives in head and neck cancer. In Cancer of the Head and Neck. Edited by Myers EN, Suen JY, Myers JN, Hanna EY. Philadelphia, PA: Saunders; 2003:1-5.

18. Bonner JA, Harari PM, Giralt J, et al.: Radiotherapy plus cetuximab for squamous-cell carcinoma of the head and neck. $\mathrm{N}$ Engl J Med 2006, 354:567-578.

19. Lentsch EJ, Myers JN: Pathogenesis and progression of squamous cell carcinoma of the head and neck. In Cancer of the Head and Neck. Edited by Myers EN, Suen JY, Myers JN, Hanna EY. Philadelphia, PA: Saunders; 2003:5-28.

20. Iyer AK, Khaled G, Fang J, Maeda H: Exploiting the enhanced permeability and retention effect for tumor targeting. Drug Discov Today 2006, 11:812-818.

21. Goel R, Shah N, Visaria R, et al.: Biodistribution of TNF-alphacoated gold nanoparticles in an in vivo model system. Nanomed 2009, 4:401-410.

22. Pirollo KF, Chang EF: Does a targeting ligand influence nanoparticle tumor localization or uptake? Trends Biotechnol 2008, 26:552-558.

23. Yguerabide J, Yguerabide EE: Light-scattering submicroscopic particles as highly fluorescent analogs and their use as tracer labels in clinical and biological applications. I. Theory. Anal Biochem 1998, 262:137-156.

24. El-Sayed I, Huang X, Macheret F, Humstoe JO, et al.: Effect of plasmonic gold nanoparticles on benign and malignant cellular autofluorescence: a novel probe for fluorescence based detection of cancer. Technol Cancer Res Treat 2007, 6:403-412.

25. El-Sayed IH, Huang X, El-Sayed MA: Surface plasmon resonance scattering and absorption of anti-EGFR antibody conjugated gold nanoparticles in cancer diagnostics: applications in oral cancer. Nano Lett 2005, 5:829-834.

26. Sokolov K, Follen M, Aaron J, et al.: Real-time vital optical imaging of precancer using anti-epidermal growth factor receptor antibodies conjugated to gold nanoparticles. Cancer Res 2004, 63:1999-2004.

27. Li PC, Wang CR, Shieh DB, et al.: In vivo photoacoustic molecular imaging with simultaneous multiple selective targeting using antibody-conjugated gold nanorods. Opt Express 2008, $16: 18605-18615$.

28. Zharov VP, Lapotko DO: Photothermal imaging of nanoparticles and cells. IEEE J Selected Topics Quant Elect 2005, 11:733-751.

29. Nikoobakht B, Wang J, El-Sayed MA: Surface-enhanced Raman scattering of molecules adsorbed on gold nanorods: off-surface plasmon resonance condition. Chem Phys Lett 2002, 366:17-23.

30. Wang H, Huff TB, Zweifel DA, et al.: In vitro and in vivo twophoton luminescence imaging of single gold nanorods. Proc Natl Acad Sci U S A 2005, 102:15752-15756.

31. Song KH, Kim C, Maslov K, Wang LV: Noninvasive in vivo spectroscopic nanorod-contrast photoacoustic mapping of sentinel lymph nodes. Eur J Radiol 2009, 70:227-231.

32. Agarwal A, Huang SW, O'Donnell M, et al.: Targeted gold nanorod contrast agent for prostate cancer detection by photoacoustic imaging. J Appl Phys 2007, 102:064701-064701-4.

33. Niidome T, Akiyama $Y$, Shimoda $K$, et al.: In vivo monitoring of intravenously injected gold nanorods using near-infrared light. Small 2008, 4:1001-1007.

34. •• Qian X, Peng XH, Ansari DO, et al.: In vivo tumor targeting and spectroscopic detection with surface-enhanced Raman nanoparticle tags. Nat Biotechnol 2008, 26:83-90. This article demonstrates detection of tumor in vivo using SERSs.

35. Aaron J, Nitin N, Travis K, et al.: Plasmon resonance coupling of metal nanoparticles for molecular imaging of carcinogenesis in vivo. J Biomed 2007, 12:034007.

36. Kneipp J, Kneipp H, Kneippa K: SERS - a single-molecule and nanoscale tool for bioanalytics. Chem Soc Rev 2008, 37:10521060 . 
37. Oyelere AK, Chen B, Huang X, et al.: Peptide conjugated gold nanorods for nuclear targeting. Bioconjugate Chem 2007, 18:1490-1497.

38. Huang X, El-Sayed IH, Qian W, El-Sayed MA: Cancer cells assemble and align Gold nanorods conjugated to antibodies to produce highly enhanced, sharp and polarized surface Raman spectra: a potential cancer diagnostic marker. Nano Lett 2007, 7:1591-1597.

39. Weissleder R: A clearer vision for in vivo imaging. Nat Biotechnol 2001, 19:316-317.

40. Loo C, Lowery A, Halas NJ, et al.: Immunotargeted nanoshells for integrated cancer imaging and therapy. Nano Lett 2005, 5:709-711.

41. Hirsch LR, Stafford RJ, Bankson JA, et al.: Nanoshell-mediated near-infrared thermal therapy of tumors under magnetic resonance guidance. Proc Natl Acad Sci U S A 2003, 100:13549-13554.

42. Schwartz JA, Shetty AM, Price RE, et al.: Feasibility study of particle-assisted laser ablation of brain tumors in orthotopic canine model. Cancer Res 2009, 69:1659-1667.

43. Jain PK, Lee KS, El-Sayed IH, El-Sayed MA: Calculated absorption and scattering properties of gold nanoparticles of different size, shape, and composition: applications in biological imaging and biomedicine. J Phys Chem B 2006, 110: 7238-7248.
44. Huang X, Jain PK, El-Sayed IH, El-Sayed MA: Determination of the minimum temperature required for selective photothermal destruction of cancer cells with the use of immunotargeted gold nanoparticles. Photochem Photobiol 2006, 82:412-417.

45. Lapotko D: Therapy with gold nanoparticles and lasers: what really kills the cells? Nanomed 2009, 4:253-256.

46. Akiyama Y, Mori T, Katayama Y, Niidome T: The effects of PEG grafting level and injection dose on gold nanorod biodistribution in the tumor-bearing mice. J Control Release 2009, 139:81-84.

47. Hainfeld JF, Dilmanian FA, Slatkin DN, Smilowitz HM: Radiotherapy enhancement with gold nanoparticles. J Pharm Pharmacol 2008, 60:977-985.

48. Diagaradjane P, Shetty A, Wang JC, et al.: Modulation of in vivo tumor radiation response via gold nanoshell-mediated vascular-focused hyperthermia: characterizing an integrated antihypoxic and localized vascular disrupting targeting strategy. Nano Lett 2008, 8:1492-1500.

49. Peng XH, Qian X, Mao H, et al.: Targeted magnetic iron oxide nanoparticles for tumor imaging and therapy. Int $\mathrm{J}$ Nanomedicine 2008, 3:311-321.

50. Wang $\mathrm{C}$, Chen J, Talavage T, Irudayaraj J: Gold nanorod/Fe3O4 nanoparticle "nano-pearl-necklaces" for simultaneous targeting, dual-mode imaging, and photothermal ablation of cancer cells. Angew Chem Int Ed Engl 2009, 48:2759-2763. 